\title{
Breast ultrasound in the age of advanced technology and artificial intelligence
}

\author{
Kazunori Kubota ${ }^{1}$
}

Received: 14 February 2021 / Accepted: 22 February 2021

(c) The Japan Society of Ultrasonics in Medicine 2021

Breast diagnostic imaging has been undergoing changes as of late. The main reasons for these changes are the introduction of artificial intelligence (AI) into clinical practice and the introduction of simple diagnostic approaches that utilize the latest diagnostic imaging technology. Against this backdrop, I pondered the significance of ultrasound in diagnostic imaging of the breast going forward.

The prognosis of breast cancer, which is the most commonly diagnosed cancer among women, can be improved with early detection and appropriate treatment. Breast ultrasound is one of the most commonly used modalities for the detection and diagnosis of breast cancer, but it has two major problems: it is operator dependent and it has low reproducibility. As such, it has not been employed for mass screenings despite the fact that the diagnostic ability of ultrasound has been reported to exceed that of mammography [1].

Contrast-enhanced magnetic resonance imaging (MRI), which is recommended for diagnostic imaging of the population at high risk of breast cancer, has been shown to have a higher diagnostic ability than mammography and ultrasound combined [2]. MRI follow-up of hereditary breast and ovarian cancer (HBOC) after onset has been covered by health insurance in Japan since 2020. Recently, the usefulness of screening with breast MRI was demonstrated in women with dense breasts and women in the normal-risk population [3]. A scanning technique called abbreviated breast MRI is advocated for screening purposes [4]. With this technique, which consists of only a single early-phase contrast-enhanced series, a judgment is made based fundamentally on only a single maximum intensity projection (MIP) image. The benefits include short scan times and simple image interpretation, and adoption of this technique has begun worldwide. Another technique being investigated is ultrafast dynamic

Kazunori Kubota

kubotard@dokkyomed.ac.jp

1 Department of Radiology, Dokkyo Medical University Saitama Medical Center, Saitama, Japan
MRI, which consists of several early-phase scans taken at intervals of $6 \mathrm{~s}$ or less. Breast MRI, with its short scan times, simple image interpretation, and high diagnostic ability, is likely to become the first choice for screening in general.

Another major revolution taking place is that in the sphere of AI. Software that utilizes deep learning is now available on the market and is poised to change the nature of diagnosis. Use of AI in diagnostic imaging in clinical practice is very important as it saves time, alleviates physician and radiologist fatigue, and compensates for lack of experience and skill. We have also investigated AI, and we found that it was possible to create AI with high diagnostic ability using high-quality training data [5]. It is possible to distinguish between benign and malignant lesions and detect lesions with high precision at the image level using today's AI, but it cannot render assessments based on such factors as patient background, clinical course, and laterality, and it cannot make an overall judgment.

I would like to emphasize the following three messages based on an understanding of the current situation and a review of the role of ultrasonography:

1. Comprehensive and reproducible imaging studies The issue of reproducibility is on its way to being overcome thanks to the emergence of automated whole-breast ultrasound. However, it will take time for medical institutions to adopt it due to the high cost, and the problem of the labor intensiveness of image interpretation remains. Going forward, it will be important to gather evidence that demonstrates that it is possible to perform studies without missing anything by means of serial scanning using hand-held ultrasound devices.

2. Simple diagnostic approaches The fundamental goal of diagnosis is to divide a lesion into one of three categories (suspicious, probably benign, and benign) based on its composition and morphology. A lesion that has been assessed as probably benign can be further divided into a suspicious lesion requiring biopsy or a lesion requir- 
ing follow-up by combining the primary findings with secondary image findings (e.g., additional morphology information, blood flow, stiffness). It is important to be proficient at diagnosis based on the various guidelines, but diagnostic imaging that enables one to make sound management decisions is ultimately needed.

3. Implementation of AI based on an understanding of its characteristics I think that AI can be used both for guaranteeing that the diagnosis is comprehensive and guaranteeing the quality of the diagnosis. In the case of ultrasound, it may be difficult to display the whole breast on a single image like MRI, but I think that AI can be applied to produce the most comprehensible images possible.

Ultrasound is an examination that patients can undergo with peace of mind without the invasiveness associated with radiation exposure, drug administration, and so forth. Ultrasound is a modality that can be used for screenings, for detailed examinations, and to guide biopsies, and its diagnostic ability is expected to improve even further in the future. Therefore, I hope that breast ultrasound-related research and medical care are pursued in a forward-looking manner while keeping an eye on trends in other modalities, so that advanced technology is used for patient-friendly medicine.

\section{References}

1. Ohuchi N, Suzuki A, Sobue T, et al. Sensitivity and specificity of mammography and adjunctive ultrasonography to screen for breast cancer in the Japan Strategic Anti-cancer Randomized Trial (J-START): a randomised controlled trial. Lancet. 2016;387:341-8.

2. Kuhl C, Weigel S, Schrading S, et al. Prospective multicenter cohort study to refine management recommendations for women at elevated familial risk of breast cancer: the EVA trial. J Clin Oncol. 2010;28:1450-7.

3. Mann RM, Kuhl CK, Moy L. Contrast-enhanced MRI for breast cancer screening. J Magn Reson Imaging. 2019;50:377-90.

4. Kuhl CK, Schrading S, Strobel K, et al. Abbreviated breast magnetic resonance imaging (MRI): first postcontrast subtracted images and maximum-intensity projection-a novel approach to breast cancer screening with MRI. J Clin Oncol. 2014;32:2304-10.

5. Fujioka T, Mori M, Kubota K, et al. The utility of deep learning in breast ultrasonic imaging: a review. Diagn (Basel). 2020;10:1055.

Publisher's Note Springer Nature remains neutral with regard to jurisdictional claims in published maps and institutional affiliations. 